\title{
Interstellar transmitter concept (King David's Sling)
}

\author{
Glen Monahan ${ }^{1}$, Sarvraj Singh ${ }^{2}$ \\ ${ }^{1}$ BA (Philosophy), Memorial University of Newfoundland, Canada \\ ${ }^{2}$ Jaypee University of Engineering and Technology, MP, India
}

\section{Email address:}

glenemonahan@gmail.com (G. Monahan)

\section{To cite this article:}

Glen Monahan, Sarvraj Singh. Interstellar Transmitter Concept (King David's Sling). American Journal of Modern Physics. Vol. 2, No. 3 , 2013, pp. 138-143. doi: 10.11648/j.ajmp.20130203.17

\begin{abstract}
A simple operating principle (similar to a combination of a pottery wheel and a catapult or, simply, the weapon used by David to slay Goliath) coupled with the success of some moderate engineering challenges may allow for the transmission of a carrier wave from Earth to Mars in less than one second!This paper also directly addresses the controversy of light speed variance/invariance (which has arisen from the "wave/particle nature of light" debate) by referencing Joseph A. Rybczyk’s 2012 paper, "Lunar Laser Evidence of Light Speed Variance”.
\end{abstract}

Keywords: Interstellar Transmitter, Faster than Light, FTL, Operating Principle, Epistemology, Relativity, Tachyon

\section{Introduction}

It's not necessarily apparent that the sling David used could not have beena "sling-shot" style weapon. But a little internet research into the history of rubber bears out sucha weapon in ancient times would be extraordinarily unlikely."When Christopher Columbus revisited Haiti on his second voyage, he observed some natives playing ball. Columbus' own men had brought their Castilian wind-balls to play with in idle hours. However, they found that the balls of Haiti were incomparably superior toys; they bounced better. These high bouncing balls were made from a milky fluid, the consistency of honey, which the natives harvested by tapping certain trees and then cured over the smoke of palm nuts" [1]. And it was centuries later whence a U.S. circuit court"established for all time the claim of the American, Charles Goodyear, to be the sole inventor of vulcanized rubber" [2]. Oneshould also note that, even if the ancients could have tooled a high-powered, hi-tech, hunting style sling-shot in part by utilizing some long-lost rubber formula, one may expect such a device to make its way into sufficiently popular distribution as to render it detectable to modern archaeology.

The Bible tells us David's sling launched missile hit Goliath with such force that "the stone sank into his forehead" [3]. It must have been propelled with a respectable momentum. One can quite plausibly envisage the velocity component of this force originating from several accelerating turns of a rope attached to a piece of cloth (or leather) that cradles a stone. The only thing missing would be the tremendous skill (quite literally, the trick of the wrist) to release the stone at full throttle and with great accuracy. One can attribute this phenomenal skill to divine intervention or Zen; the type of Zen often used in reference to an elite NBAforward's scoring ability.

Now let's switch gears for a moment. Utilizing a piece of wax to demonstrate the mind alone (and not the imagination spurred on by the senses) is the inspector of truth, René Descartes established clarity and distinctiveness [4] as an epistemologicalhallmark and (notably), in doing so, is often credited (how much credit he actually deserves may be a bit of a contentious topic in many academic circles) with fuelling the scientific revolution that brought on the Industrial Age.

So, it is from within this aforementioned context of God and/or Zen that I present this otherwise technical paper with the following caveat:

As far as I can fathom, it contains no clear and distinct contradiction with respect to the Relativity Principle.

Here is the link for "The principle of relativity; original papers by A. Einstein and H. Minkowski. Translated into English by M.N. Saha and S.N. Bose; with a historical introd. by P.C. Mahalanobis (1920)" [5].

And for what I consider to be an all-inclusive exposition of relativity and what Einstein himself calls "... a few happy hours of suggestive thought!" [6],please see [7].

\section{Operating Principle}

Now, let's begin with an ordinary household electric drill. 
In general, they are said to reach a rotational speed of 2400 rotations per minute (RPMs). To the best of my knowledge, I believe a relatively recent applicationof brushless electric motor technology (induction motor) has given us the Dremel or rotary tool ["Similar rotary tools (for example, those sold by Sears under their Craftsman brand) are also sometimes colloquially called dremels in an example of a genericized trademark."[8]]. The dremel reaches speeds of 35,000RPMs This is a little bit better than a magnitude ten (10) improvement in rotational speed.

If we consider the conditions of outer space (visa vie its close-to-zero gravity and vacuum-like air resistance), we can easily see the potential for several of these magnitude ten improvements (MTIs) in rotational speed. If we can obtain 5 of these MTIs, we can reach 3,500,000,000 RPMs in a rotary device placed in an orbiting satellite.

A disk of about 12.5 inches in diameter gives a circumference of one (1) meter. Rotating at 3.5 billion RPMs, an emitter placed on the circumference will be travelling at the speed of.58c (c being the velocity of light). And light emitted from this point will travel at the speed of $1.58 \mathrm{c}$.

Now imagine another increase of magnitude ten in rotational speed! The result is 2.94c. And another;20.44c. And so on. At 1,945.44c, a roundtrip communiqué between Mars and Earth would take, at most, 1.25 seconds.

Table 1. Speeds of disc, circumference \& carrier wave.

\begin{tabular}{|c|c|c|}
\hline RPMs & $\begin{array}{l}\text { Speed of emitter } \\
\text { placed on } \\
\text { circumference of disk }\end{array}$ & $\begin{array}{l}\text { Speed of carrier } \\
\text { wave in kilometres } \\
\text { per second or } c\end{array}$ \\
\hline 2400 & $144 \mathrm{~km} / \mathrm{h}$ & $300,000.040$ \\
\hline 35,000 & $2100 \mathrm{~km} / \mathrm{h}$ & $300,000.583$ \\
\hline 350,000 & $5.833 \mathrm{~km} / \mathrm{s}$ & $300,005.833$ \\
\hline $3.5 * 10^{\wedge} 6$ & $58.333 \mathrm{~km} / \mathrm{s}$ & $300,058.333$ \\
\hline $3.5 * 10^{\wedge} 7$ & $583.333 \mathrm{~km} / \mathrm{s}$ & $300,583.333$ \\
\hline $3.5 * 10^{\wedge} 8$ & $5,833.33 \mathrm{~km} / \mathrm{s}$ & $305,833.333$ \\
\hline $3.5 * 10^{\wedge} 9$ & $58,333.3 \mathrm{~km} / \mathrm{s}$ & $358,333.333$ \\
\hline $3.5 * 10^{\wedge} 10$ & $583,333 \mathrm{~km} / \mathrm{s}$ & $2.94 \mathrm{c}$ \\
\hline $3.5 * 10^{\wedge} 11$ & $5.8 * 10^{\wedge} 6 \mathrm{~km} / \mathrm{s}$ & $20.44 c$ \\
\hline $3.5 * 10^{\wedge} 12$ & $5.8 * 10^{\wedge} 7 \mathrm{~km} / \mathrm{s}$ & $195.44 c$ \\
\hline $3.5 * 10^{\wedge} 13$ & $5.8 * 10^{\wedge} 8 \mathrm{~km} / \mathrm{s}$ & $1,945.44 \mathrm{c}$ \\
\hline $3.5 * 10^{\wedge} 14$ & $5.8 * 10^{\wedge} 9 \mathrm{~km} / \mathrm{s}$ & $19,445.44 c$ \\
\hline
\end{tabular}

\section{The Experiment}

Skepticsmay claim that, for Einstein, the speed of light is constant and so cannot be speeded up by adding the speed of the emitter.

According to Max von Laue, the relativity principle claims "From the totality of natural phenomena, one can determine with increased approximation and increased exactitude, a reference system $\mathrm{x}, \mathrm{y}, \mathrm{z}, \mathrm{t}$ in which the natural laws are valid in certain, mathematically simple forms. This reference system is, however, in no way uniquely defined by the phenomena. There is rather a three-times infinite manifold of equally valid systems, mutually moving with uniform velocity" [9].

Not to denigrate Laue's synopsis of the relativity principle, I do find many claiming that this is the proof that light, being a natural phenomenon, has a constant velocity. To me, all it claims is that each reference system moves at a constant velocity. For instance a planet moving around a sun moves at a constant velocity, a solar system moving around a red giantmoves at a constant velocityand the universe(the manifold of all systems) moves at a constant velocity (though what the universe might be moving through is certainly unknown).

This (my claim as to what the relativity principle means) would explain dark regions in space as simply star systems moving away from us at a speed greater than light.

And what else would explain the following?

Once upon a time, a man travelling at twice the speed of sound on the Concordcalled ahead to the passenger in front of him. Now sound is a natural phenomenon and its speed is $343 \mathrm{~m} / \mathrm{s}$. But in order for the passenger in the forward seat to hear the man, the sound must have been travelling at $1029 \mathrm{~m} / \mathrm{s}$ relative to anyone standing still on the earth.

Not yet convinced? I propose the following empirical experiment to test my understanding of the relativity principle:

One or two transmittersof the design in figure 1 are sent to the International Space Station (ISS). The speeded-up (or not) carrier wave is bounced of the surface of the moonand back to the ISS. Thence, the time interval of the signal's roundtripis measured.

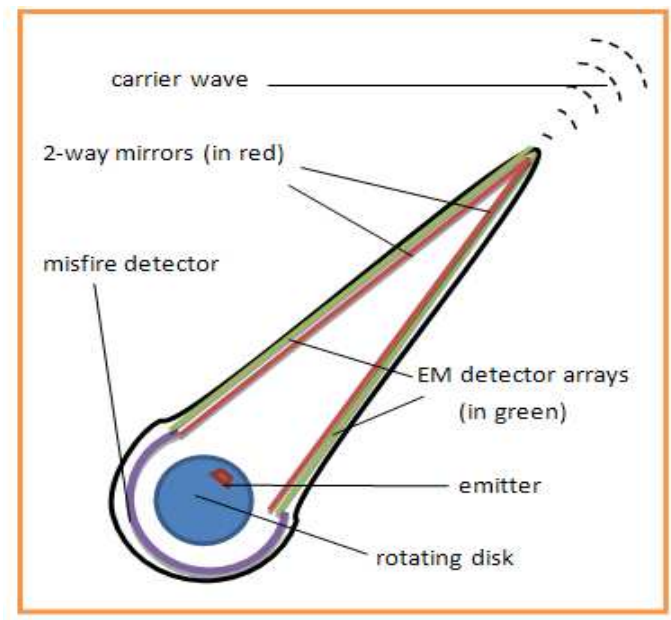

Figure 1. Interstellar transmitter. 


\section{Further Evidence (Meaningful Ref- erences and Work Based on Scientific Principles)}

But to go through the expense of sending equipment into orbit to test the idea of a layman like myself might be, to say the least, a little suspect and/or risky.

Joseph A. Rybczyk's 2012 paper, "Lunar Laser Evidence of Light Speed Variance" may serve to mitigate these concerns. Here is the paper's abstract:

"Correct formulas are derived for use in interpreting the results of the NASA lunar laser test of the invariance of light speed. The subject formulas appear to confirm that the speed of light is affected by the relative motion of the light source"'[10].

\section{Engineering Challenges}

1) Problem: Any EM carrier wave emitted from a rotating device cannot be expected to travel consistently concentrated in a straight line from the emitter to the distant receiver as the reference origin of the wave (the edge of the rotating disk) is in motion.

Solution (fig. 1): The emitting side of the satellite houses a funnelling window directed at the distant receiver. The funnel has two walls; an inside wall and an outside wall. The inside wall of the funnel is made up of a two-way mirror substrate. Placed on the inside of the outside wall are a series of EM detectors. These detectors can signal adjustments to the timing of emitting, thus keeping the signal firing relatively straight and, in doing so, may also mitigate the possible problem of a later emission getting ahead of an earlier one (should the earlier one make initial contact close to the start of the funnel and, thereby, bounce back and forth inside the funnel for a time).

2) Problem: Non-EM or unknown source interference.

Solution: Simple digital modulation (channel switching) of little or no greater complexity of that employed when experiencing what is colloquially known as the "schizophrenic C/D" computer storage drive problem.

3) Problem:Considering signalling being our main agenda with significant reception of the message, the introduction of noise is indispensable either in the form of interplanetary dust or absorption by water vapour molecules (in case of signals arising from stars).

According to Shannon's Classical Theory for rate of information transmitted, considering all possible multi-level and multi-phase encoding techniques, the channel capacity $\mathrm{C}$ (in bits/sec), and data that can be sent with a given average signal power S subjected to Additive White Gaussian Noise of power $\mathrm{N}$, in an analogue channel is:

\section{$\mathrm{C}=\mathrm{B} \log _{2}(1+\mathrm{S} / \mathrm{N})($ Shannon-Hartley theorem $)$}

From quantum physics we know that the energy per carrier photon is equal to hv joules, where $\mathrm{h}$ is Planck's constant. Hence, we calculate that the maximum theoretical photonic information transmission efficiency $\varepsilon_{\gamma}$ is:

$$
\varepsilon_{\gamma}=\left\{\log _{2}(1+\mathrm{S} / \mathrm{N})\right\} / \mathrm{h} \text { bits/joule-second }
$$

As far as our bandwidth of the transmitted signal is considered, the channel capacity would be inexplicable.

Solution: Modifications in analogue modulation techniques shall serve the purpose of reducing bandwidth.

\section{An Alternative Emitter}

Being a bit retrospective (i.e. "But on second thought, perhaps light is a particle and not a wave"), the emitter at the edge of the rotating disk may be replaced by a light source such as an atom, molecule, orNano crystal.Upon transition from the excited state to ground state, packets of light (contextually, a quanta of energy $\mathrm{E}=\mathrm{h} v$ ) will be emitted which would be equivalent to the difference of energies of the two states of the photon states. The phase of the photon in spontaneous emission is random as is the direction in which the photon propagates. The constructive or the destructive interference patterns between the quanta (emitted as a result of spontaneous emission at the tip of the transmitter)in addition to the carrier wave itself can act as unique codes for particular transmitters and receivers operating at different carrier waves. This can be quite useful in interstellar cryptography.

\section{Food for Thought [Metaphysical Walkie-talkie (MPWT)]}

“(A)tachyonic particle is a hypothetical particle that alwaysmoves faster than light. ...Most physicists think that faster-than-light (FTL) particles cannot exist because they are not consistent with the known laws of physics" [11].Just because FTL particles may not be consistent with the known (or even all possible to come) laws of physics, it does not necessarily follow that they do not exist. Though they may indeed never exist in even our most studious observations of the 3-D physical world, they are intuitively part and parcel of the metaphysical single dimensional and 2-D worlds.

In Euclidian geometry, two points define a line. If we think of these two points of the line as being single dimensional particlesakin to quantum singularities ("In factsingularities are infinitely small according to relativity" [12]) then one can possibly fathom how each of these particles can exist in the infinite expanse of the lines they define; thereby having the capacity to traverse the infinite breadth of space in a zero-time interval.However, "Euclid described a line as "breadthless length", and introduced several postulates as basic unprovable properties from which he constructed the geometry" [13]. In practical terms, two points will never define a very accurate line. When an experienced carpenter uses penciled points to set up a line to draw a cutting reference for sawing, he will pencil in three or more points. At best, in practical terms, two points can only define a curve.So perhaps two points define a circle or, rather, an 
infinite circular plane.

Now, posita somewhat different, though aesthetically similar, transmitter (fig. 2).Its rotating disk emitter shoots out a photon at a velocity greater than the speed at which the photon itself is supposed to travel. And suppose this action causes the photon to condense into a qualia singularity [I'll term this a qualia singularity since the arbitrarily described phenomenon has all the characteristics (qualities) of a quantum singularity save the quanta, quantum or mass of energy]. Further, suppose all but the tip of the transmitter can be encased in a field that contains the qualia singularity and the singularity is directed out thru the tip of the transmitter.

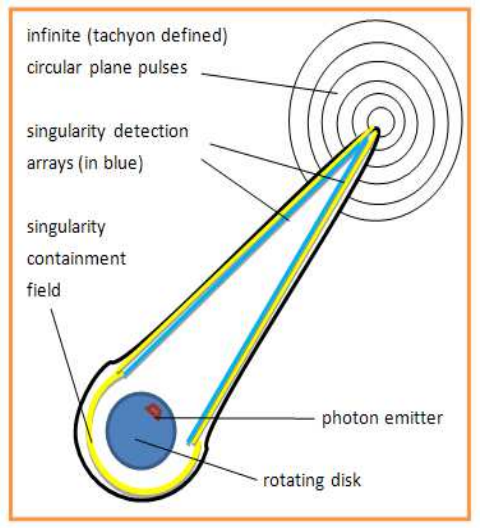

Figure 2. MPWT transmitter.

Now, try to envision the dilemma of the qualia singularity. Having exited the transmitter's field and being infinitesimally small, it is alone in a vast expanse of (relative to the singularity - it being infinitely tiny) nothingness.In response, it splits into two tachyons. And these two tachyons now exist in the infinite circular plane they define.

Juxtaposing two such transmitting devices (fig. 3) irrespective of the orientation would generate twosets of tachyonic circular planes. From these a skeletal (as it is without substance) 3-D spherical wave frontwillperhaps be generated at the place where the two planes intersect (provided that the transmitters are not in the same plane which could only occur for a brief instant in the worst of circumstances) in a manner similar to that of theHuygens's Principle.

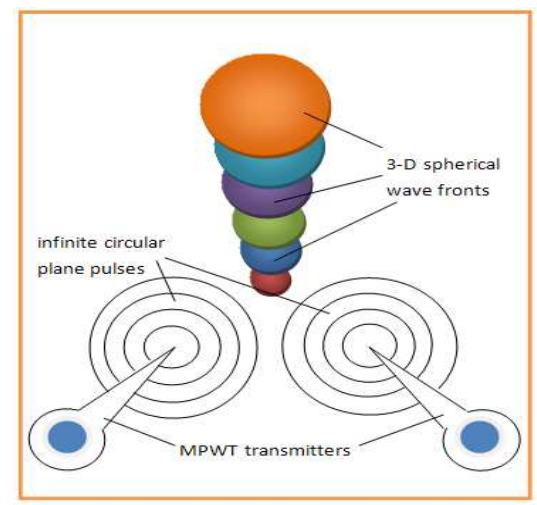

Figure 3.Juxtaposed MPWT transmitters.
Remarkably, this 3-D spherical wave has the advantage of being able to pass unhindered thru all physical space. It might possibly be hindered by the transmitter's containment field or other such devices but likely little or nothing else would interfere with the transmission. Further posit this instantaneous, ubiquitous transmission can be received by an FTL rotating funnel receiver (fig. 4) by means of condensing the two pairs of tachyons into a pair of quantum singularities which expand into photons as they are brought from the FTL edge of the funnel to the sub-light interior.One notable disadvantage is each reception would destroy that part of the transmission.

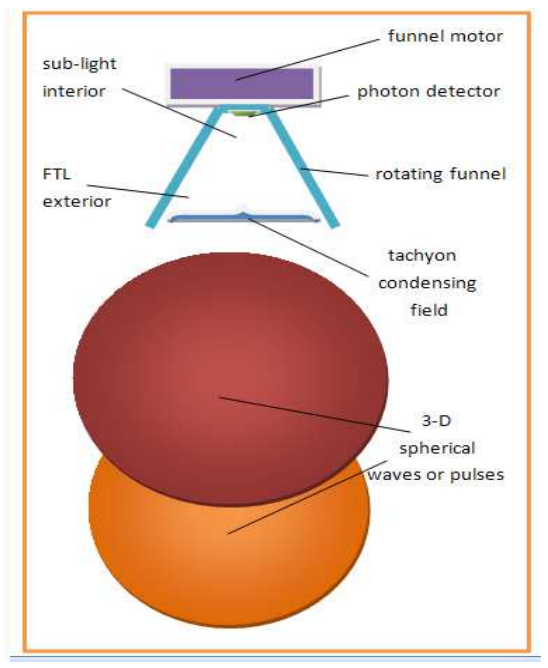

Figure 4.MPWT receiver.

Regarding this unsubstantial 3-D spherical wave (or pulse) I should note that, according to Einstein, Descartes found extension without substance to be unfathomable. Einstein writes "One can understand that it was repugnant to Descartes to consider space as independent of material objects, a thing that might exist without matter" [14].

\section{Method}

Firstly, I believe Einstein has been quoted (or perhaps audio recorded) as saying the universe is the laboratory of the cosmic scientist. As an educated layman, I submit that the "big picture" which is critical to an understanding of the universe (the ultimate big picture) is more easily accessible to me than a trained and/or network PhD physicist. Experts of the like are often described as those who know more and more about less and less; hardly a state conducive to an understanding of the big picture.

But foremost, I'd like to take this opportunity to pay homage to Tyrone Lai for his epistemology (scientific method); the CryptanalyticalTheory of Discovery. As I recall, the basic components are:

1) Educated Guess Method - hypothesis

2) Method of Empirical Testing - experiment

3) Method of Progressive Evaluation(MPE) - verification/refutation 
Now let's expand on this last component, the MPE ("method of progressive evaluation" [15]). The idea is: if you can move on in your scientific investigation, then you have evidence that you are on the right track - your method of knowing (i.e. your epistemology) is working; your theory, for the time being, is verified. If you cannot move on then your theory is being refuted. In the former case you are productive and in the latter you'd be "spinning your wheels" to continue on with the same theory. Karl Popper expounded a similar view regarding the validity of a scientific theory being tied to the ability of being able to refute the theory. He writes "the criterion of the scientific status of a theory is its falsifiability, or refutability, or testability." [16]

To put all this in perspective by way of the example of observing a dark region is space - do we describe it as a quantum singularity (black-hole) and scour the universe for the associated "exotic matter" to fuel a warp bubble or do we hypothesis what we have in the dark region is a star system moving away from us at a speed greater than light? The latter passes the refutability test i.e. it is capable of being refuted. The former will never pass the refutability test (at least, it will never pass the MPE) as its arbitrarily described nature denies empirical testing. By further example, do we build the interstellar transmitter (fig. 1) while paying all due respect to proprietary telecommunication technology or do we do foreseeably endless design on the MPWT of figures 2-4? Think about it. What say you?

\section{Last Word (Dedication)}

Come on people! For land sakes' [Here, I coin a new version of the idiom land sakes or lands sakes. I choose this new plural possessive form,land sakes', to reflect the often perceived existential necessity (and hence, need for possession) of land claims for Homo sapiens.], wake-up and smell the coffee. There's a potential bean plantation or two in a nearby solar or star system. Guaranteed.

\section{The Obligatory Conclusions}

Yes, the interstellar transmitter (aka King David's Sling) of figure 1 is the way to go. Meanwhile, the MPWT of figures 2-4 is just a waste of time.

So who's interested in the universe of work that's available outside the Earth-Moon system for those utilizing the real-time controls \& communications courtesy of FTL? Well not me, that's for sure. As I've previously stated, I'm a layman. And laymen are simply too lazy to work. Why do you think we're called laymen? We layaround a lot, that's why.

\section{Epilogue}

You don't have to go far to find out about science or even epistemology for that matter. David Suzuki (whilst under contract with the Canadian Broadcasting Company (CBC)) will happily inform that for every question science answers, you are left with ten more questions. So am I being facetious in asking the following two questions? :

1) Will any of Commander Hadfield's myriad of mechanical experiments yield a disk's circumference based emitter (or something of the sort) that will have travelled faster than the speed of light?

2) And, if the answer to 1) is yes, will we all be left waiting for Sarah Brightman to sing us the results?

Again, am I facetious in asking those two questions? You tell me.

\section{Acknowledgements}

Dr. Tyrone Lai, whose patient tutelage in his Cryptanalytical Theory of Discovery was indispensable and integral to my university education.

Dr. Alan MacPherson (and his better-half, Dr.Joyce MacPherson) who, within the confines of Memorial University Career Experience Program (MUCEP) grants, imparted to me key graduate experience whilst an under-grad.

And a special thank-you to the administration, staff \& other patronsof the Gander Public Librarywho all made me feel at home and (eventually) learned to live with me.

\section{References}

[1] Available online:http://inventors.about.com/cs/inventorsalphabet/a/rubbe r_2.htm.

[2] Available online:http://inventors.about.com/cs/inventorsalphabet/a/rubbe r.htm.

[3] Samuel 17:49, The Holy Bible, New King James Version. Nashville: Nelson. 1982. ISBN 978-0840700537 (This ISBN was found on Wiki, not the Bible itself.).

[4] René Descartes, Meditations on First Philosophy, (old page numberings) pp. 30-31.

[5] Available online:http://archive.org/details/theprincipleofre00einsuoft

[6] Albert Einstein (1962), Relativity: The Special and the General Theory, Crown Publishers Inc., New York, p. 5.

[7] Albert Einstein (1962), Relativity: The Special and the General Theory, Crown Publishers Inc., New York.

[8] Available online: http://en.wikipedia.org/wiki/Dremel.

[9] Available online: http://en.wikisource.org/wiki/The_Principle_of_Relativity_( Laue_1913).

[10] Available online: http://www.mrelativity.net/LunarLaserEvidenceofLightSpee dVariance/Lunar\%20Laser $\% 20$ Evidence $\% 20$ of $\% 20$ Light $\% 20 \mathrm{Sp}$ eed\%20Variance.htm

[11] Available online: http://en.wikipedia.org/wiki/Tachyon. 
[12] Available http://en.wikipedia.org/wiki/Quantum_singularity.

[13] Available http://en.wikipedia.org/wiki/Line_(geometry)\#Ray.

[14] Albert Einstein (1962), Relativity: The Special and the General Theory, Crown Publishers Inc., New York,p.137.

online:

online:
[16] Karl Popper (2004), Conjectures and Refutations: The Growth of Scientific Knowledge, London: Routledge. ISBN 0-415-28594-1, circa p.37-38. 